\title{
L'hypogénitalisme du pharaon akhénaton. Une énigme historique
}

\author{
G. ANDROUTSOS \\ Fondation Internationale Hippocratique de Cos, Grèce \\ 1, rue Ipirou, 104 33, Athènes, Grèce
}

\section{RÉSUMÉ}

\begin{abstract}
Akhénaton est le plus fascinant des pharaons égyptiens. Ses représentations montrent d'étranges particularités (caractères féminins, crâne allongé, hydrocéphalie, acromégalie) qui sont peut-être symboliques, à moins qu'elles ne témoignent de quelque maladie hormonale. Cet article présente les principales hypothèses concernant la maladie du roi hérétique.
\end{abstract}

Mots clés : Akhénaton, syndrome de Frölich, lipodystrophie progressive, acromégalie, hydrocéphalie, homosexualité

\section{INTRODUCTION}

A près de $300 \mathrm{kms}$ au sud du Caire, sur la rive orientale du Nil, dans la plaine d' Amarna s'est joué un des grands drames de l' ancienne Égypte. Dans les premières années du XIXe siècle, les archéologues européens qui se rendirent sur ces lieux constatèrent sur les reliefs des scènes dans lesquelles les figures royales étaient dessinées différemment du style traditionnel de l' Égypte ancienne. Le roi (et dans une moindre mesure la reine) était représenté comme si sa tête était déformée, avec un long nez, des lèvres épaisses, un menton proéminent et un cou sinueux. Son aspect physique, de plus, était distinctement féminin, avec ses seins lourds, ses hanches courbes et ses cuisses amples. La question se posait de savoir si c' était un roi et sa compagne, ou bien deux reines, l'une d'elles travestie en pharaon.

\section{INTERPRÉTATIONS}

En 1894, le savant anglais Petrie [18] put confirmer qu' Aménophis IV et Akhénaton n'étaient qu'une seule et même personne, laquelle avait épousé une reine, Néfertiti. Il écarta également les suggestions plus anciennes de Mariette [16], selon lesquelles Akhénaton était un eunuque ou une femme travestie. Il pensait que de telles théories étaient formellement contredites par la vie conjugale du roi: celui-ci apparaissait en effet à côté de son épouse sur tous les monuments, il l'embrassait en public et la cajolait tandis qu'elle était assise sur ses genoux. Le savant anglais rappela aussi que le famille du souverain s'accroissait régulièrement et il tenta de montrer que les aspects physiques d' Akhénaton et Néfertiti étaient intrinsèquement différents. On ne peut toutefois affirmer que ce dernier jugement soit pleinement convaincant. En outre Petrie prétendit que les particularités des traits du visage d'Akhénaton étaient héritées d'une mère asiatique. Mais ses comparaisons se fondaient sur une identification erronée.

\section{LA PATHOLOGIE}

Plusieurs diagnostics ont été proposés pour la maladie dont souffrait Akhénaton: ils ne peuvent s'appuyer que sur les représentations figurées car la momie de la tombe 55 de la Vallée des Rois, longtemps considérée comme celle d'Akhénaton, est en réalité celle de son frère et co-régent Sémenkharê: cette restitu- 
tion est fondée sur des données anthropométriques et paléopathologiques [12].

Les premiers chercheurs qui accédèrent à Amarna et à ses tombes pensèrent que la figuration de roi que l'on voyait sur les reliefs était celle d'une femme et qu'on avait donc représenté deux reines agissant de concert. Akhénaton est en effet rendu sur ces reliefs avec le même cou allongé, les mêmes hanches larges, les mêmes seins gonflés et les mêmes cuisses grasses que Néfertiti. Comme il porte à l'occasion une longue robe collante semblable à un vêtement de femme, les images du roi ont souvent été confondues avec celles de la reine et vice versa. Le caractère féminin de la morphologie d'Akhénaton est bien illustré par les torses provenant des statues brisées trouvées près du temple d' Amarna. Il est tout à fait impossible de décider sur les seuls critères anatomiques si ces torses sont ceux du roi ou de la reine. Le savant français Eugène Lefébure, par exemple, s'appuyant sur le précédent notable de la reine Hatchepsout, a émis il y a un siècle l' hypothèse qu'Akhénaton était en fait une femme costumée en homme [21].

Outre le fait que les représentations d'Akhénaton le montrent efféminé ou androgyne, elles soulignent aussi certaines déformations qui n'appartiennent ni à des hommes normaux ni à des femmes normales. Ces caractères anormaux, dépeints sous une forme exagérée, ont permis à un certain nombre de spécialistes de diagnostiquer chez le sujet ainsi représenté des désordres du système endocrinien, et plus spécialement un mauvais fonctionnement de la glande pituitaire.

\section{Akhénaton souffrait - il d'un syndrome de Babinski - Fröhlich?}

Le syndrome de Babinski-Fröhlich fut décrit d'abord par M.J. Babinski (Rev. neurol. Paris, 1900, 8, 531), puis par A. Fröhlich (Wien. Klin., Rdsch., 1901, 15, 883). Ce terme a été souvent utilisé abusivement pour désigner l'adiposité physiologique prépubertaire ou pubertaire (Syn: syndrome adiposo-génital, s. hypophysaire adiposo-génital, $\mathrm{s}$. adiposohypogénital (Langeron), s. de Frohlich, s. de LaunoisCléret, s. hypophyso-thalamique, dystrophie adiposo-génitale, s. de Langeron). Ce syndrome est en rapport avec une atteinte de l'hypophyse ou de la région infundibulo-tubérienne: infection, tumeur hypophysaire (adénome chromophobe, craniopharyngiome).

Dans le syndrome de Fröhlich l'adiposité peut varier en degré, mais il y a une distribution de graisse typiquement féminine dans les zones de la poitrine, de l'abdomen, du pubis, des cuisses et des fesses. Les membres inférieurs cependant sont maigres et les jambes ressemblent à des "pantalons de golf". A un stade précoce du mal, il peut y avoir une suractivité passagère de la glande pituitaire, qui peut produire de telles distorsions du crâne avec une croissance excessive de la mâchoire, mais ce stade est suivi d'un autre, où l'on observe un sous fonctionnement de la glande et de l'hypogonadisme. Le diagnostic du syndrome de Fröhlich ne peut se faire que lorsque le patient qui a atteint l'âge de la puberté ne se développe pas normalement, que sa voix reste haut perchée, que son poil n'apparaît pas et que ses organes sexuels restent au stade infantile. Comme les tumeurs de cette glande sont rares avant la puberté, le désordre ne peut commencer qu'au moment de l'adolescence. Un stade ultérieur de la maladie est le gonflement des seins, de l'abdomen, des fesses et des cuisses. Un symptôme concomitant peut être l'hydrocéphalie. Il est étrange que l'"expressionnisme" (comme on l'a souvent qualifié) introduit par Akhénaton dans l'art égyptien ait représenté la figure humaine comme si elle exhibait jusqu'à un degré exagéré les anomalies de quelque désordre endocrinien. Comme c' est l'image du roi qui montre ces caractères de la manière la plus marquée, on est autorisé à penser que celui-ci a souffert du syndrome de Fröhlich et qu'il a souhaité se voir représenté avec toutes les déformations qui le différenciaient du reste de l'humanité.

L'une des statues de Karnak à l'effigie du roi le représente nu et complètement asexué (Musée du Caire, No 55938). C' est à son propos que Elliot Smith [19] , en 1907, a évoqué le syndrome de Babinski-Fröhlich. L'absence d'organes génitaux externes ne peut être considérée ici comme un caractère pathologique en faveur du début de l'affection à un âge prépubertaire. En effet, on a supposé qu'un pagne métallique 
pouvait être prévu, ou tout au moins un devanteau; on peut accepter également le fait que le roi se soit fait représenter en Osiris asexué. Par contre, l'adiposité rétro-mammaire qu' on retrouve sur d'autres figures du pharaon, l'abdomen volumineux, lourd, les fesses arrondies, les cuisses enrobées de graisse contrastant avec des jambes normales seraient autant de caractères compatibles avec le diagnostic de syndrome adiposo-génital, encore que les bras ne soient pas gros [14] (Figure 1).

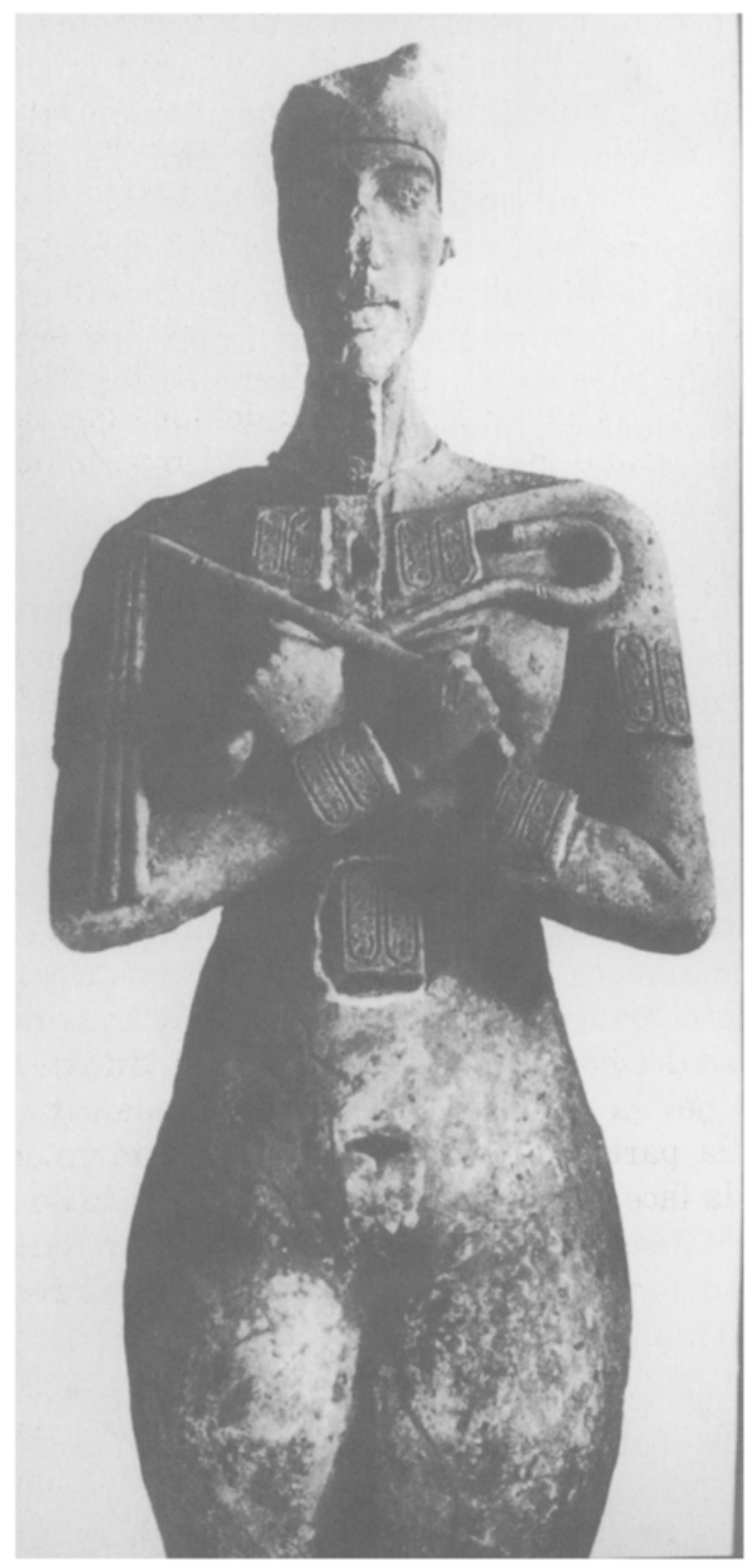

Figure l: Akhénaton ayant l'aspect d'un eunuque, ce qui suggère que celui-ci aurait souffert de quelque désordre endocrinien. Colosse en provenance de Karnak. (Musée égyptien, Le Caire).
Il y a cependant un sérieux obstacle qui empêche d'attribuer à Akhénaton un syndrome de Fröhlich et qui a interdit aux spécialistes d'être plus catégoriques dans leur diagnostic. Akhénaton est le seul parmi les pharaons à s'être fait représenter comme un père de famille [2]. Comment donc un époux si conjugal et un père si attaché à sa progéniture pourrait-il avoir souffert de ce syndrome de Fröhlich, qui l'aurait rendu nécessairement stérile et dénué de libido? Les six princesses sont désignées dans chaque cas comme "la fille du roi, née de son corps, née de la grande épouse Néfertiti"; cela laisse supposer que leur père est bien Akhénaton, même si d'autres candidats ont été suggérés, comme Aménophis III qui pourtant ne peut être impliqué à moins d'avoir eu une longue corégence avec son fils. Il y a d'un autre côté, des témoignages qui montrent que vers la fin $\mathrm{du}$ règne d'Akhénaton, lorsque les six filles ont atteint l'âge nubile, celui-ci a eu des enfants d'elles. Même si on a aussi suggéré que c' était le corégent Sémenkharê qui en était le père, des considérations chronologiques rendent la chose hautement improbable. Ces relations incestueuses ne sont pas inconnues ailleurs dans les familles de rois divins, mais la paternité d'Akhénaton, bien qu'elle ait été niée par certains savants, semble suffisamment claire. De plus, les textes figurant sur les talatates d'Hermopolis et se référant à "la favorite" et à la "très aimée" Kiya ont révélé que cette autre reine avait également eu une fille d'Akhénaton. Une telle puissance procréatrice de la part du roi ne dénote pas précisément un manque de virilité. En dépit de l'exagération avec laquelle il encouragea ses artistes à représenter sa personne, il ne peut avoir souffert d' une anomalie suffisamment chronique pour compromettre sérieusement sa vigueur sexuelle [20].

Enfin Adler signale, sur un calcaire trouvé à El Amarna, une ébauche du visage d' Akhénaton portant une barbe de plusieurs jours. La présence de ce caractère sexuel secondaire, s' il n' est pas une création artificielle de l' artiste, viendrait ruiner définitivement le diagnostic de syndrome de Frohlich [1].

\section{Akhénaton était -il acromégale?}

Le diagnostic d'acromégalie est fondé sur l'aspect très particulier du profil du visage avec 
l'ouverture excessive de l'angle de la mâchoire, le prognathisme et la taille considérable des oreilles (Figure 2). Selon K. Michalowski "le crâne est de forme ovoïde, la mâchoire est

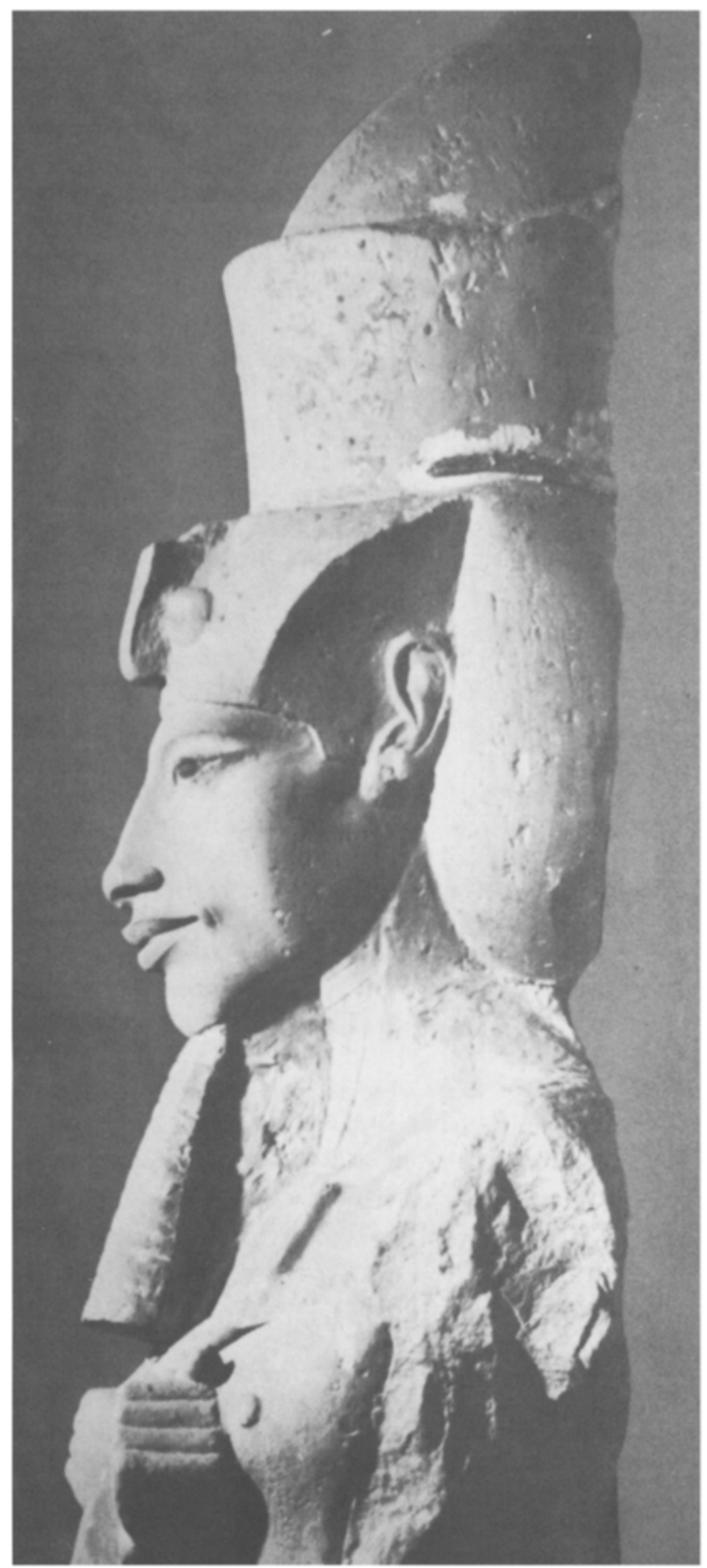

Figure 2 : Détail d'une statue d'Akhénaton, en provenance du temple d'Aton, à Karnak (Musée égyptien, Le Caire). longue presque chevaline, le cou est maigre, les bras grêles et tombants, le ventre proéminent". Acromégalie? L'aspect physique du pharaon est évocateur [13]. Paul Ghaliounghi [9] est frappé aussi par le curieux physique d'Akhénaton, par ses seins volumineux, par l'asexualité apparente de quelques statues; il conclut à un hypogonadisme survenu après la naissance des quatre filles que lui donna la belle reine Néfertiti. Alors? Acromégale et eunuque Akhénaton? Plus vraisemblablement acromégalie avec une gynécomastie car l'association des deux éléments est fréquente. Le problème ne sera résolu que si les archéologues découvrent un jour le corps d'Akhénaton, l'examinent et le radiographient [3]. Toutefois il est aussi possible que les traits d'Aménophis IV correspondent à un aspect "acromégaloïde" familial. En effet, un autre frère du roi, le prince Sémenkharê est représenté sur un bas-relief avec les mêmes défauts anatomiques que son frère: crâne ovale, maxillaire allongé, ventre proéminent [13].

\section{Le diagnostic des neurologues}

Dès 1920, M. Ameline et P. Quercy [4], neurologues français, se sont penchés sur le problème de la disproportion manifestée par la constitution physique d'Akhénaton. Sur la foi d' effigies du pharaon, ils diagnostiquèrent une lipodystrophie progressive ou maladie de Barraquer-Simons. Le même diagnostic fut posé aussi par Mackenzie-van der Noordan [15]. Il s'agit d'une affection rare, décrite par A. Simons (Zschr. Neurol. 1911, 5, 29), caractérisée par la fonte du tissu adipeux sous-cutané de la partie supérieure du corps, notamment de la face (faciès voltairien) et l'adiposité de la moitié inférieure (stéatopygie fessière, adiposité des cuisses et des jambes). Les sujets atteints de cette maladie ont bien les joues creusées par la disparition de la boule graisseuse de Bichat, le cou maigre et long, les bras décharnés, contrastant avec une pseudo hypertrophie du bassin et des membres inférieurs. (Syn: maladie de Simons, maladie de Smith, maladie de Hollander-Simons).

La majorité des savants certifient même qu'Akhénaton était atteint d'hydrocéphalie. Il est vrai que, sur la foi de rapports d'autopsie et 
d'expériences faites sur les animaux, psychiatres, neurologues et neurochirurgiens sont arrivés à des conclusions différenciées sur la nosographie de l'hydrocéphalie. De nos jours on peut examiner les malades à l'aide des radiographies, du scanner, etc., et on observe ainsi des formes très diverses d'hydrocéphalie. Aussi cette maladie n'est-elle plus considérée comme une maladie très déterminée, mais comme symptôme de diverses maladies. D'après Hans Kehrer, psychiatre et neurologue de Munster, les hydrocéphales manifestent des tendances anormales dans leur comportement psychique et social [20]. En outre, ils restent en général petits de taille et meurent jeunes. D'un autre côté, l'examen clinique montre très souvent que certaines formes de comportement ou certaines capacités seulement sont détruites ou hypertrophiées. Une irritabilité émotionnelle d'une intensité exceptionnelle est également considérée comme symptôme typique d'hydrocéphalie. Emotivité et irascibilité sont très souvent causées par des migraines très fortes, voire intolérables, qui entraînent souvent des vomissements. Or, c' est à l'époque d'Akhénaton que, pour la première fois dans d'histoire de l'art égyptien, on représente des gens en train de vomir, phénomène qui, plus tard, disparaît complètement. Les vomissements pathologiques du souverain ont-ils à ce point préoccupé le peuple qu'il les a même représentés dans ses œuvres d'art?

Donc, à partir de sa seizième année, l'époux de Néfertiti fut atteint d'une grave maladie, et, progressivement, son aspect physique devint tellement pitoyable qu'il finit par dépendre du soutien et de l'énergie spirituelle d'une forte personnalité... Néfertiti. "Outre les troubles de l'intelligence, écrit le neurologue Kehrer, il arrive souvent que les hydrocéphales présentent encore de grands changements dans d'autres fonctions psychiques. Trente-trois de nos propres malades atteints de fortes dilatations des ventricules cérébraux, présentaient des symptômes d'inertie, d'hébétude, d'apathie, d'états simili-dépressifs, mais, à l'occasion aussi, d'euphorie niaise, d'agitation constante, et d'excitabilité excessive" [21].

L'hérédité de l'hydrocéphalie est discutée. Mais il existe des observations d'après les- quelles cette maladie est congénitale et liée au sexe. Avec l'âge, Aménophis III manifesta un comportement de plus en plus apathique, et il est possible qu'il ait transmis cette maladie à son fils. Dès les années 1950, des neurologues britanniques et français ont constaté que l'apparition de l'hydrocéphalie dans la jeunesse était également liée à des troubles sexuels, tels par exemple "l' infantilisme hormonal", "la puberté précoce", et "la dystrophie adiposogénitale". Pour Akhénaton il s'agit sans aucun doute d'une régression et d'une malformation des organes génitaux [5].

L'état pathologique dans lequel Akhénaton a choisi de se faire représenter est partagé à un moindre degré par toute sa famille et tout son entourage. Il faut soupçonner que la physionomie particulière d'Amarna fut la forme idéale que les artistes reçurent instruction d'adopter, et il devint "chic" pour les proches et la famille du roi d'adopter les mêmes particularités. Il est probable qu'Akhénaton possédait un crâne platycéphale plutôt inhabituel; mais si la représentation des crânes des princesses n'est qu'un reflet de l'idéal dont Akhénaton avait décidé qu'il devait être la figuration par excellence du crâne royal, alors cela va bien au-delà de l'inhabituel. La forme exagérée des crânes des princesses est telle que l'on peut se demander légitimement si celles-ci, ou bien le modèle d'après lequel elles furent représentées, ne souffraient pas d'une forme d'hydrocéphalie [3].

Pour chacun des diagnostics proposés, on retrouve des signes positifs. Aucun cependant n'est pleinement acceptable et il faudrait admettre plutôt qu'Akhénaton a souffert d'un syndrome hypothalomo-hypophysaire complexe. Il est toutefois risqué d'affirmer l'existence d'une maladie à partir d'une représentation artistique où se mêlent la convention, l'idéalisme et une part de réalisme dont on ne peut juger l'importance [6].

\section{L'HOMOSEXUALITÉ D'AKHÉNATON}

A partir d'une petite stèle inachevée d'Amarna, qui montre Akhénaton en compagnie de Sémenkharê et en tête à tête intime pendant un repas (Figure 3), John Harris soutient une théorie percutante: pour lui, le per- 


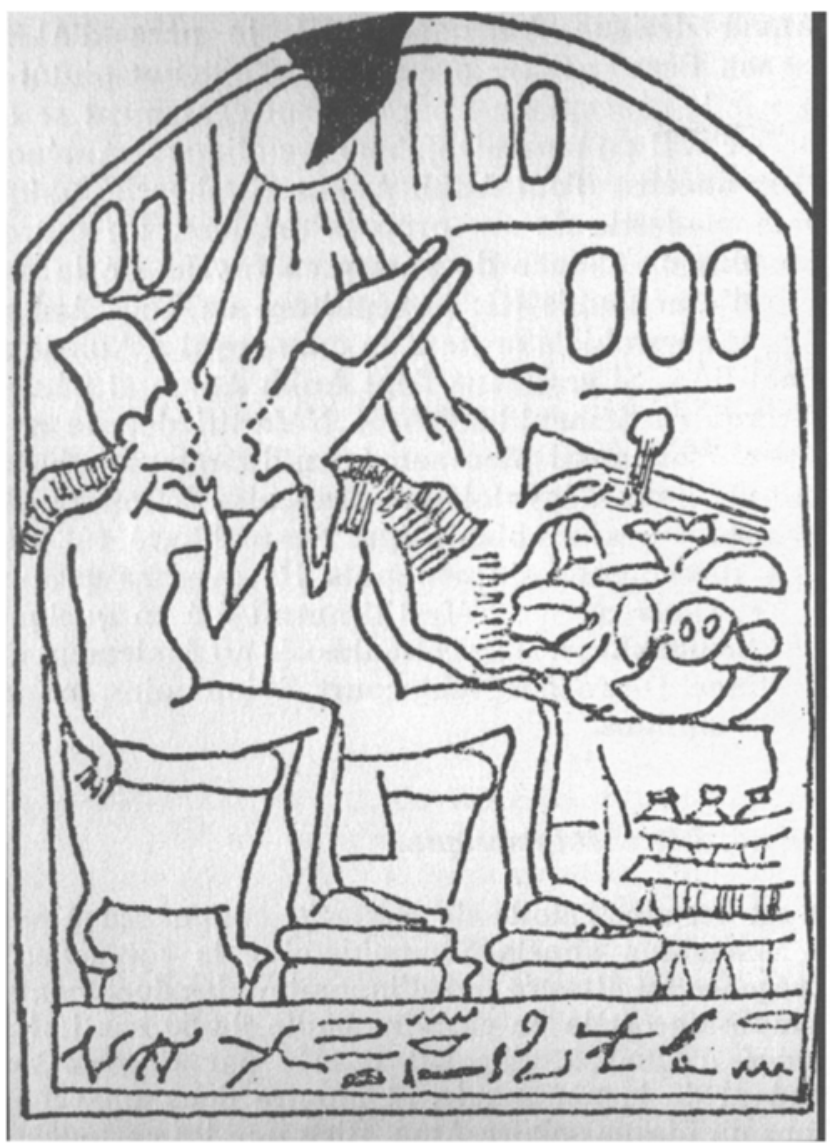

Figure 3 : La stèle 17813 échauffa les esprits des égyptologues. Akhénaton (à droite) est en train de caresser Sémenkharê (à gauche). (Musée de Berlin Charlottenbourg).

sonnage de Sémenkharê, que nous avons considéré jusqu'à présent comme le corégent d'Akhénaton, ne serait autre que... Néfertiti [11]. Quoi qu'il en soit, Sémenkharê changea son nom après l'an 14 du règne d'Akhénaton en Néfernéférou-Aton, "Celui qui est aimé d'Akhénaton". Pour Harris, " l'homme" Sémenkharê n'a jamais existé, et ce fut Néfertiti qui, dans la douzième année du règne d'Akhénaton, changea son nom en celui de Sémenkharê. Mais, malheureusement, cette hypothèse, laisse un trop grand nombre de questions sans réponses. Si jamais l'hypothèse sensationnelle de Harris se révélait exacte, la signification historique de Néfertiti s'en trouverait revalorisée; car vu sous cet aspect, l'hypothèse de Harris hisse la belle Néfertiti sur le trône, à côté de son époux, en tant que "pharaon régnant”. Quelques années plus tard Percy Edward Newberry [17] protesta contre l'hypothèse de Harris et souligna que les deux personnages, représentés sur la stèle d' Amarna, portent une couronne royale, la couronne bleue et la couronne double, privilège accordé au seul pharaon. Donc, poursuivit Newberry, cette scène ne peut représenter qu' Akhénaton et Sémenkharê. Nous concluons donc qu'Akhénaton a traité effectivement Sémenkharê comme son "épouse" et l'a donc fait représenter nanti des attributs féminins. L'attitude du bras de la momie de Sémenkharê en est un indice indiscutable; elle correspond à celle d'une momie royale féminine: le bras gauche sur la poitrine, le bras droit tendu le long du corps [8].

Les historiens Joseph, Sexte Jules l'Africain et Eusèbe accordent tous trois à Akhénaton un temps de règne de douze ans. Pour tous les trois, il est... la fille du pharaon Aménophis III [11]. Jusqu'à présent, les égyptologues repoussèrent la théorie de ce "changement de sexe" car ce qui ne doit pas être ne peut pas être. Pourtant, rien que le fait qu'une telle confusion soit unique dans tout le catalogue des rois égyptiens devrait faire réfléchir. Si nous commençons par supposer qu'Akhénaton n'était pas une femme, il faudrait rendre en premier lieu responsables de cette métamorphose tardive du pharaon les nombreuses représentations picturales sur lesquelles il ressemble plus à une femme qu'à un homme. Akhénaton s'est peut-être décidé aussi à cette démarche exceptionnelle de faire monter un de ses proches parents sur le trône pharaonique à côté de lui, parce qu'il savait parfaitement que, par suite de sa maladie, il ne pourrait plus avoir de successeur mâle [21].

De toute façon les trois dernières filles de Néfertiti ne sont pas considérées comme des enfants d'Akhénaton, par la majorité des historiens: mais on peut aussi douter qu'il revendique la paternité de Méritaton, la fille aînée. Le mariage de Sémenkharê, le corégent, avec Méritaton, la fille aînée de Néfertiti, pourrait s' expliquer aisément si Aménophis III était le père de Méritaton. Méritaton serait alors l'héritière légitime du trône, et son mariage avec Sémenkharê une démarche politique tendant à préserver la dynastie. A l'encontre de l'opinion de Hornung, il semble cependant plus vraisemblable que la mariage de Sémenkharê avec 
la fille de Néfertiti ait eu lieu avant l'accession au trône du corégent, donc dans la treizième année du règne d'Akhénaton, mais cela n'explique toujours pas, malgré tout, si le mariage fut décidé pour des motifs de tactique politique, ou pour permettre à Akhénaton de garder Sémenkharê près de lui afin de satisfaire ses tendances homosexuelles. En tout cas, sur les représentations figuratives de cette époque, nous voyons Sémenkharê en tête à tête intime aussi bien avec Méritaton qu' avec Akhénaton [7].

Sémenkharê était peut-être sexuellement ambivalent. On ne peut douter qu'il ait entretenu des relations homosexuelles avec Akhénaton, étant donné les représentations picturales conservées au Staatliche Museen de Berlin et qui montrent les deux hommes dans une attitude très tendre. Roberg Silverberg, continue à douter, malgré tous les résultats de la recherche historique, de la liaison homosexuelle des deux pharaons; il présume qu'Akhénaton pourrait être le père de Sémenkharê qui serait le fils d'une concubine quelconque. On objectera que parmi les nombreuses représentations picturales d'Akhénaton, on ne trouve aucun indice permettant de conclure que le roi avait un fils [10].

\section{LES DERNIÈRES ANNÉES D'AKHÉNATON}

Si l'on met en ordre toutes les données, on aboutit au scénario suivant pour les dernières années d'Akhénaton: vers l' an 14, Néfertiti disparaît de la scène. Après la mort de celle-ci, l' attention se porte sur une autre reine, Kiya; elle reçut le titre familier de "la favorite" avec l'épithète "la très aimée". Quoique femme de roi, elle n'était pourtant pas décrite comme une "héritière" ou une "grande épouse". D'ailleurs d'autres épouses secondaires d'Akhénaton ont également laissé des traces de leur existence, comme une certaine Ipy, "ornement royal" et "véritable favorite" du roi. Aucune cependant n'est aussi importante que Kiya. Il se pourrait que les privilèges exceptionnels dont elle bénéficiait venaient de ce que, en plus d'une fille, elle avait donné au roi un fils ou des fils. Kiya mourut à peu près vers la même époque. Même avant le décès de Néfertiti, les relations entre Akhénaton et ses filles aînées avaient pris un caractère sexuel, comme le montre la mort de Maketaton; il faut souligner pourtant que cela n'était pas le résultat d'un comportement libidineux, mais constituait un ensemble d'obligations de mise dans le cercle divin. Dans ce qui pourrait ressembler à une insistance frénétique sur d'incestueux "droits de seigneur", Akhénaton devint apparemment le père d'au moins deux filles par l'entremise des princesses Méritaton et Ankhesenpaaton, durant les trois ou quatre dernières années de son règne. Le pharaon avait besoin d'une postérité masculine pour s'assurer que sa lignée divine serait perpétuée. Dans les faits, pourtant, il y eut des déceptions dans la mesure où les filles royales ne donnèrent naissance qu'à d'autres filles.

Les décès de Tiyi, de Maketaton, de Néfertiti, de Kiya et de Méritaton, et probablement d'autres filles et petites-filles plus jeunes, ne sont probablement pas des coïncidences. Ils semblent plutôt la conséquence de l'épidémie de peste qui régnait à cette époque en dehors de l'Egypte, dans le Levant. Les mesures qu'il était possible de prendre pour les hommes de l'âge du Bronze récent afin de faire face à ce fléau se réduisaient presque exclusivement à des incantations magiques.

$\mathrm{Au}$ milieu de problèmes familiaux et politiques, Akhénaton mourut dans des circonstances qui restent totalement obscures. Les Egyptiens ne faisaient pas un événement tragique ou héroïque de la mort de leurs rois: ils étouffaient plutôt l' événement en y voyant un triomphe du mal sur le bien. Nous n'avons aucun compte rendu officiel de la fin d'Akhénaton, qui eut lieu probablement après les vendanges en sa dix-septième année de règne.

\section{REFERENCES}

1. ADLER C.: Akhénaton, le pharaon mystique 1 vol., Tallandier, Paris, 1969, 28-40.

2. ALDRED C.: Akhénaton. Roi d' Égypte, Éd. du Seuil, Paris, 1997, 78-90.

3. ALDRED C., SANDISON A.T.: The pharaon Akhénaton. A problem in egyptology and pathology, 
4. AMELINE M., QUERCY P.: Akhénaton, Rev. Neurol., 1920, 5, 448.

5. ANDROUTSOS G., MARKETOS SP.: La sexualité dans l'ancienne Égypte, Progrès en Urologie, 1994, 4/6, 715-725.

6. ANDROUTSOS G. : Les mœurs sexuelles dans l' Égypte pharaonique, Sexologies, 1997, VI, 23/48-59.

7. DAUMAS F.: "La civilisation de l' Égypte pharaonique", Arthaud, Paris, 1965, 492.

8. DERRY D.E.: Note on the skeleton hitherto believed to be that of king Akhénaton, Ann. Serv., 31, 1931, 119 .

9. GHALIOUNGUI P.: A medical study of Akhénaton, Ann. Serv., 47, 1947, 29.

10. GHALIOUNGUI P.: La médecine des pharaons, Robert Lafont, Paris, 1983, 174.

11. HARRIS J.R.: Akhénaton or Néfertiti?, Acta Orientalia, 38, 1977, 5.

12. HARRISON R.G.: An anatomical examination of the pharaonic remains purported to be Akhénaton, JEA, 52,1966, 95.

13. HAZARD J., PERLEMUTER L.: L'homme hormonal. Une histoire illustrée, Hazan, Paris, 1995, 345346.

14. LECA A. P.: La médecine égyptienne au temps des pharaons, Roger Dacosta, Paris, 1971, 169-174.

15. MACKENZIE - VAN DER NOORDAN M.C.: Un aspect médical de la parenté entre Akhénaton, Sémenkharê et Toutankhamon, Organorama, 1965, 3-12.

16. MARIETTE A.: Les papyrus Égyptiens du musée de Boulaq, vol. 1, Paris, 1871, 32-34.

17. NEWBERRY P.E.: King Ay, the successor of Tut' ankhamûn, JEA, No 18 (1932), 50.

18. PETRIE W.M.F.: Tell el-Amarna, Londres, 1894, 13, 93-95.

19. SMITH G.E.: The Royal Mummies, CCG 61051-100, Le Caire, 1912.

20. SNORRASON E.: Cranial deformation on the reign of Akhénaton, Bull. Hist. Med., XX, 5, 601-610.

21. VANDENBERG PH.: Néfertiti. La première biographie archéologique, Pierre Belfond, Paris, 1976, 4855 .

\section{Pharaoh' s Akhenaten hypogenitalism. An historical enigma \\ G.ANDROUTSOS}

Akhenaten is the most fascinating of the Egyptians pharaohs. His representations show some strange particularities (feminization, cranial deformation, hydrocephalus, acromegaly) which have, eventually, a symbolic character unless they suggest the existence of an endocrinous disorder.

Through this paper, we try to elucidate the most probable diagnosis of Akhenaten' s illness.

Key-words : Akhenaten, Frolich's syndrom, progressive lipodystrophy, acromegaly, hydrocephalus, homosexuality. 